\title{
Ultrafast Charge Transfer of a Valence Double Hole in Glycine Driven Exclusively by Nuclear Motion
}

\author{
Zheng Li, ${ }^{1,2,3, *}$ Oriol Vendrell, ${ }^{1,2, \dagger}$ and Robin Santra ${ }^{1,2,4}$ \\ ${ }^{1}$ Center for Free-Electron Laser Science, DESY, Notkestraß e 85, D-22607 Hamburg, Germany \\ ${ }^{2}$ Hamburg Centre for Ultrafast Imaging, Luruper Chaussee 149, D-22761 Hamburg, Germany \\ ${ }^{3}$ SLAC National Accelerator Laboratory, Menlo Park, California 94025, USA \\ ${ }^{4}$ Department of Physics, University of Hamburg, Jungiusstraße 9, D-20355 Hamburg, Germany \\ (Received 14 June 2015; revised manuscript received 13 August 2015; published 2 October 2015)
}

\begin{abstract}
We explore theoretically the ultrafast transfer of a double electron hole between the functional groups of glycine after $K$-shell ionization and subsequent Auger decay. Although a large energy gap of about $15 \mathrm{eV}$ initially exists between the two electronic states involved and coherent electronic dynamics play no role in the hole transfer, we find that the double hole is transferred within 3 to $4 \mathrm{fs}$ between both functional ends of the glycine molecule driven solely by specific nuclear displacements and non-Born-Oppenheimer effects. The nuclear displacements along specific vibrational modes are of the order of $15 \%$ of a typical chemical bond between carbon, oxygen, and nitrogen atoms and about $30 \%$ for bonds involving hydrogen atoms. The time required for the hole transfer corresponds to less than half a vibrational period of the involved nuclear modes. This finding challenges the common wisdom that nuclear dynamics of the molecular skeleton are unimportant for charge transfer processes at the few-femtosecond time scale and shows that they can even play a prominent role. It also indicates that in $\mathrm{x}$-ray imaging experiments, in which ionization is unavoidable, valence electron redistribution caused by nuclear dynamics might be much faster than previously anticipated. Thus, non-BornOppenheimer effects may affect the apparent electron densities extracted from such measurements.
\end{abstract}

The transfer of electrical charge on a microscopic scale plays a fundamental role in chemistry, in biology, and in technological applications related to energy conversion and storage [1], and is a key step in the dynamics of molecules and materials damaged by the absorption of $\mathrm{x}$-ray photons, unfolding on attosecond to femtosecond time scales [2,3]. Charge migration in molecules after valence photoionization with attosecond coherent light sources has drawn much attention in the past years [4-9]. In charge migration, a coherent superposition of electronic states of the ionized system, which might in principle be prepared by an ultrashort laser pulse of enough spectral bandwidth, leads to coherent electronic dynamics. Assuming fixed nuclei in space, time scales between 1 and 12 fs have been reported from simulations of particular coherent hole migration examples in molecules [8-10]. Owing to the availability of ultrashort light sources with sufficient bandwidth in the extreme ultraviolet regime to produce migrating holes, schemes to probe their time evolution have been put forward [10,11], and measurements of coherent hole evolution in atoms [12] and molecules [13,14] have recently been reported. Remarkably, interest in charge migration was initially sparked by measurements of charge transfer in peptides using nanosecond lasers to photoionize and probe the subsequent charge transfer [15], where the dynamics of nuclei and consequently non-BornOppenheimer effects must have played a prominent role. In particular, the non-Born-Oppenheimer coupling between nuclei and electrons is known to be crucial, even at times as short as 1 to $2 \mathrm{fs}$, if photoionization promotes the ionized molecule close to a region of conical intersection between excited electronic states of the ion $[13,16]$.

Here, we show theoretically that an ultrafast electron-hole transfer in the valence shell of an organic molecule occurring in a time scale of 3 to 4 fs can be solely driven by nuclear displacements. By this we precisely mean that no electronic coherence between the involved electronic states is imprinted on the system in the preparation step and that all nuclear and electronic dynamics of the field free system are initiated by atomic displacements. In particular, we illustrate this process for the hole transfer dynamics in doubly ionized glycine starting from electronic states with two initially localized holes in the same inner-valence orbital of either oxygen or nitrogen atoms. Such electronic states can be reached via Auger decay of core-ionized molecules. The electron holes are transferred between the two ends of the glycine molecule driven by specific nuclear displacements of a few tenths of an angstrom resulting in nuclear configurations of strong nonadiabatic coupling between electrons and nuclei. The hole transfer dynamics occur on a time scale at least $50 \%$ shorter than any of the vibrational periods of the glycine molecule in its neutral ground electronic state.

Two aspects are key for selecting initial electronic states with localized valence holes in the same inner-valence orbital. (a) Valence double-hole states can be prepared in an ultrafast manner by selective $K$-shell ionization of, e.g., 
oxygen, nitrogen, or carbon atoms with few-femtosecond or subfemtosecond $\mathrm{x}$-ray pulses [17-21]. Realistic prospects for subfemtosecond pulses at $\mathrm{x}$-ray free-electron laser facilities exist [20]. This is followed by ejection of an Auger electron on a time scale of a few femtoseconds [18] resulting in a doubly charged molecule. (b) The electronic states of the doubly charged molecule with two localized valence holes on either oxygen or nitrogen atoms are energetically separated by about $15 \mathrm{eV}$ and can be easily resolved from the kinetic energy of the corresponding Auger electron. Consequently, the experimental observation of these dynamics with femtosecond time resolution $[2,22]$ requires a probing scheme of the parent ion involving a coincident measurement of the ejected Auger electron.

In order to describe the electronic structure of the valence doubly ionized molecule we adopt a two-hole configuration-interaction $(2 \mathrm{~h}-\mathrm{CI})$ scheme, in which hole mixing is included [23]. Each dicationic state $\left|\Psi_{l}^{N-2}\right\rangle$ is expanded in the basis of two-hole configurations constructed from molecular orbitals obtained at the Hartree-Fock level for the neutral system

$$
\left|\Psi_{l}^{N-2}\right\rangle=\sum_{i \leq j}^{N_{o}} C_{i j}^{(l)}\left|\Phi_{i j}^{N-2}\right\rangle \equiv \sum_{i \leq j}^{N_{o}} C_{i j}^{(l)} \hat{c}_{i} \hat{c}_{j}\left|\Phi_{0}^{N}\right\rangle,
$$

where $\left|\Phi_{0}^{N}\right\rangle$ is the $N$-electron Hartree-Fock ground state wave function and $\hat{c}_{i}$ is the annihilation operator of the $i$ th molecular orbital. For glycine, with $N=30$ valence electrons in $N_{o}=15$ occupied orbitals this results in $N_{o}\left(N_{o}+1\right) / 2=120$ spin singlet configurations. Only spin singlet configurations are considered here as the initial localized double-hole states of interest are spin singlets. The molecular dynamics in the manifold of 120 dicationic excited states are described with the mixed quantumclassical fewest-switches surface hopping method [24]. The 2h-CI Hamiltonian is diagonalized at each time step and the corresponding potential energy surfaces, gradients, and nonadiabatic couplings necessary for the classical evolution of the nuclear coordinates and quantum evolution of the electronic degrees of freedom are computed. Further details on surface-hopping approaches can be found in Refs. [24-27]. In short, for each trajectory of the fewestswitches surface hopping ensemble the amplitude of each electronic eigenstate is integrated in time according to the Schrödinger equation

$$
i \hbar \dot{A}_{l}(t)=\sum_{m} H_{l m}(\mathbf{R}(\mathbf{t}), \dot{\mathbf{R}}(t)) A_{m}(t),
$$

where $H_{l m}$ is a matrix element of the effective timedependent Hamiltonian for the evolution of the electronic subsystem along the corresponding classical nuclear trajectory [24]. In our case this is a $120 \times 120$ matrix. The diagonal elements correspond to the adiabatic potential energy surfaces $V_{l}(\mathbf{R})$ whereas the off-diagonal elements contain the nonadiabatic couplings $-i \hbar \dot{\mathbf{R}} \cdot\left\langle\Psi_{l}^{N-2}\left|\nabla_{\mathbf{R}}\right| \Psi_{m}^{N-2}\right\rangle$ between electronic states. The electronic wave packet propagated along each nuclear trajectory hence reads $|\Psi(t)\rangle=$ $\sum_{l} A_{l}(t)\left|\Psi_{l}^{N-2}\right\rangle$. The ensemble of nuclear trajectories represents a classical approximation of the time evolution of the corresponding quantum wave packet.

Initial conditions for positions and velocities are then sampled from a thermal distribution at $300 \mathrm{~K}$. All trajectory-averaged quantities reported were obtained over 150 trajectories. The initial electronic density matrix with elements $\rho_{l m}(0)=A_{l}^{*}(0) A_{m}(0)$ for the ensemble of trajectories started in state $m$ is set to $\rho_{m m}(0) \propto I_{m}$ and zero otherwise, with $I_{m}$ the Auger intensities in Fig. 1. This corresponds to the assumption of no electronic coherence after Auger decay. Under this assumption, electronic
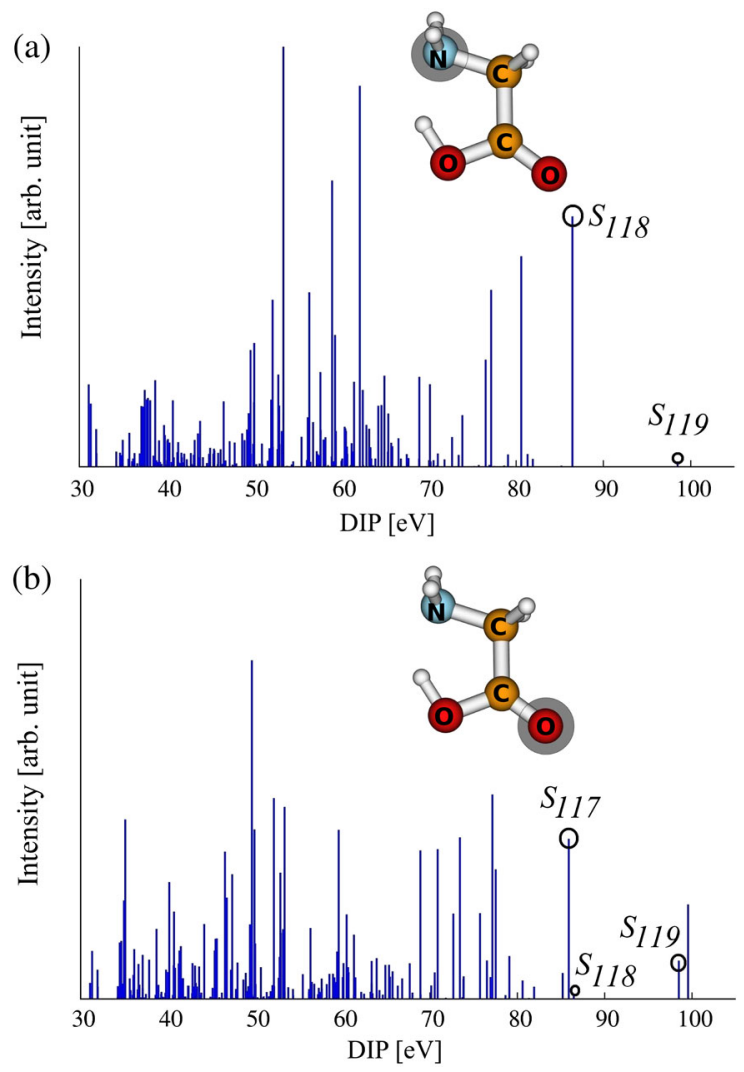

FIG. 1 (color online). Auger intensity for valence doubly ionized singlet states of glycine $\left(\mathrm{NH}_{2} \mathrm{CH}_{2} \mathrm{COOH}\right)$ after decay of a $K$-shell core hole in (a) the nitrogen atom of the amino group and (b) one of the oxygen atoms in the carboxyl group. The energy axis refers to the double ionization potential (DIP) of the corresponding electronic state at the equilibrium geometry of the neutral system. A gray circle labels the atom where the primary $K$-shell photoionization is assumed to take place. Electronic eigenstate 118 is strongly dominated by a $\left[(\mathrm{N} 2 s)^{-2}\right]$ configuration. States 119 and 120 are dominated by $\left[(\mathrm{O} 2 s)^{-2}\right]$ configurations whereas state 117 corresponds to a $2 s$ hole in each oxygen atom, $\left[(\mathrm{O} 2 s)^{-1},(\mathrm{O} 2 s)^{-1}\right]$. 
dynamics are exclusively driven by nuclear motion, since $H_{l m}=0$ for $l \neq m$ in Eq. (2) for static nuclei.

Relative Auger intensities as a function of the double ionization potential (DIP) for each dicationic eigenstate of the 2h-CI Hamiltonian were obtained by the method of population analysis [27,36-38] and are shown in Fig. 1. The DIP relates to the kinetic energy of the Auger electron as $E_{\text {kin }}=\mathrm{IP}_{c}-\mathrm{DIP}$, where $\mathrm{IP}_{c}$ is the core ionization potential for primary ionization. By inspecting the $\left|C_{i j}^{(l)}\right|^{2}$ configuration weights of states $l=119$ and $l=120$ of the 2h-CI Hamiltonian one sees that they have a strong $\left[(\mathrm{O} 2 s)^{-2}\right]$ character, i.e., two holes on the same oxygen atom [27]. These states correspond to the energetically isolated spectral lines with a DIP near $100 \mathrm{eV}$. State 118 with a DIP of $\sim 85 \mathrm{eV}$ becomes populated only when the primary photoionization occurs on the nitrogen atom. It is mostly dominated by $\left[(\mathrm{N} 2 s)^{-2}\right]$ configurations [27]. The Auger spectra in Fig. 1 are in good agreement with the calculated spectra of glycine using particle-particle Green's function techniques [39]. Because of the large energy gap of about $15 \mathrm{eV}$ between states dominated by $\left[(\mathrm{O} 2 s)^{-2}\right]$ and $\left[(\mathrm{N} 2 s)^{-2}\right]$ configurations, the electronic and nuclear dynamics initiated from the localized double-hole states at either atomic site can, in principle, be probed with complete knowledge of the initial electronic state of the ion by coincident detection of the energy of the Auger electron [40-43].

We now focus on the charge redistribution dynamics initiated after Auger decay populating an electronic state dominated by $\left[\mathrm{O}(2 s)^{-2}\right]$ configurations, namely $S_{119}$. As

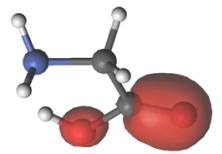

$0.00 \mathrm{fs}$
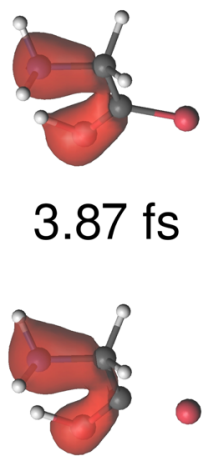

$5.81 \mathrm{fs}$
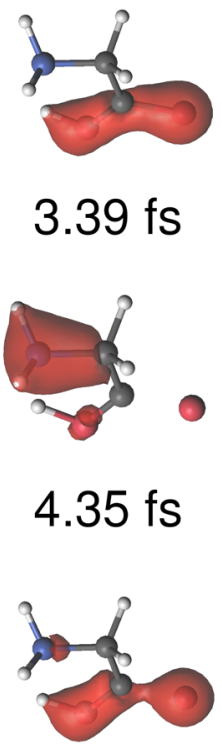

3.39 fs
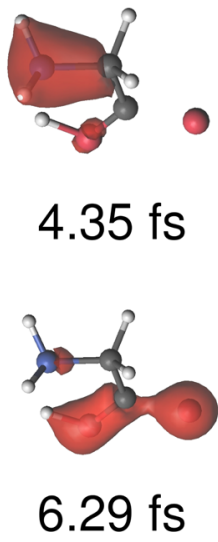

FIG. 2 (color online). Temporal evolution of double-hole charge density $Q(\mathbf{r}, t)$ of a representative trajectory initiated from state $S_{119}\left[(\mathrm{O} 2 s)^{-2}\right]$. shown in Fig. 1, the eigenstate $S_{119}$ is initially separated by an energy gap of roughly $15 \mathrm{eV}$ from state $S_{118}$, in which the two holes are localized on the nitrogen atom, meaning that the absence of nuclear motion would leave the two holes localized on the $\mathrm{COO}$ group. However, the positive charge associated with the double hole is redistributed in a very fast manner as seen in Fig. 2, where we present the double-hole density in the glycine dication for a representative trajectory. The double-hole density is defined as the difference between the electron densities of neutral and double-hole states $Q(\mathbf{r}, t)=\rho^{0}(\mathbf{r}, t)-\rho^{-2}(\mathbf{r}, t)$. The ultrafast hole transfer is illustrated in Fig. 3 by the ensembleaveraged charge $\left\langle Q_{X}\right\rangle$ on each atom type obtained from a Mulliken charge analysis [44] of the dicationic eigenstates along each trajectory. A significant amount of positive charge initially on the oxygen atoms is transferred to the nitrogen atom at the other end of the glycine backbone within $4 \mathrm{fs}$ and transferred back to the oxygen atoms within $6 \mathrm{fs}$, which is more than $50 \%$ shorter than the fastest vibrational period in neutral glycine.

Under the assumption, based on the large energy gap between the states of interest, that the Auger decay process has initially populated adiabatic electronic states, the ultrafast hole transfer must be the result of nuclear displacements connecting to a region of nonadiabatic coupling between the corresponding electronic states. Specifically, a seam of conical intersection between states $S_{119}$ and $S_{118}$ can be reached through a downhill path on the potential energy surface (PES) of state $S_{119}$ from the vicinity of the starting configuration [27,28]. In particular, the asymmetric $\mathrm{COO}$ stretch and the $\mathrm{OH}$ stretch of the carboxylic group illustrated in Fig. 4(c) are the main nuclear modes that lead to stabilization of state $S_{119}$, with both $\left[\left(\mathrm{O} 2 s^{-2}\right)\right]$ in an oxygen atom and destabilization of state $S_{118}\left[\left(\mathrm{~N} 2 s^{-2}\right)\right]$ finally closing the $15 \mathrm{eV}$ gap. The region of crossing between electronic states of $\left[(\mathrm{O} 2 s)^{-2}\right]$ and $\left[(\mathrm{N} 2 s)^{-2}\right]$ character is reached in about 3 to $4 \mathrm{fs}$ as illustrated in Fig. 4(a). After the crossing region, the adiabatic state $S_{119}$

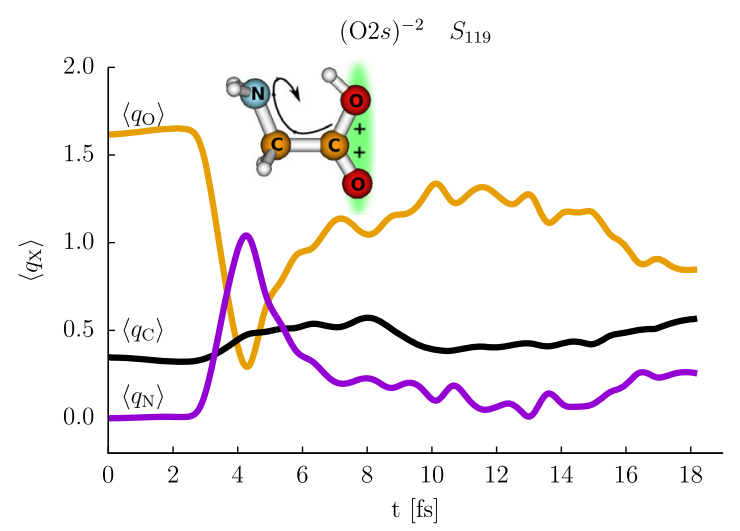

FIG. 3 (color online). Charge on heavy atoms of the glycine dication, when the dynamics are initiated from state $S_{119}\left[(\mathrm{O} 2 s)^{-2}\right]$. 

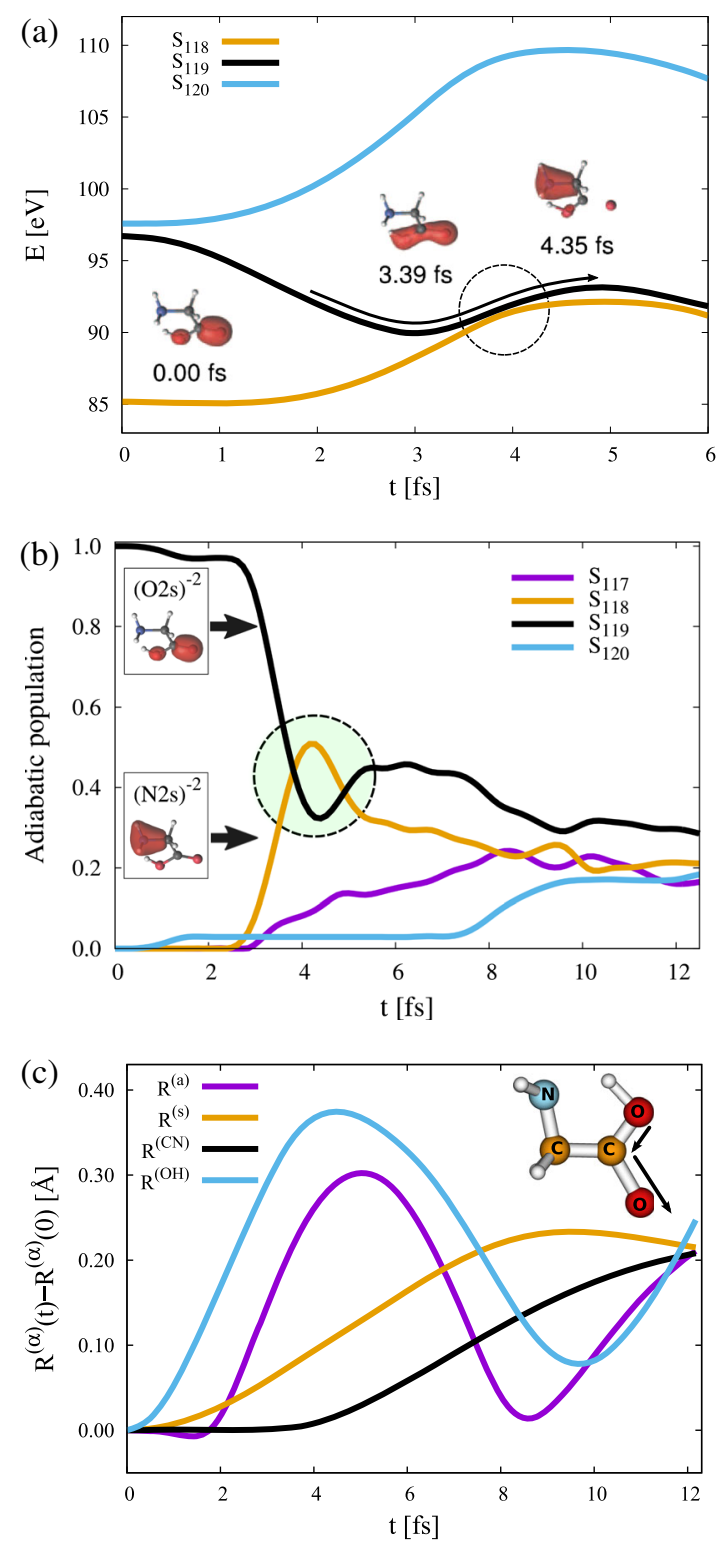

FIG. 4 (color online). (a) Potential energy surfaces of electronic states $S_{118}, S_{119}$, and $S_{120}$ along one representative trajectory started in state $S_{119}$. The evolution of the full set of 120 potential energy surfaces along this trajectory is shown in Fig. S1 [27]. The circle indicates a region of avoided crossing between the two potential functions. (b) Ensemble averaged adiabatic populations. (c) Ensemble averaged asymmetric COO stretch $R^{(\mathrm{a})}=\left|R_{\mathrm{C}-\mathrm{OH}}-R_{\mathrm{C}=\mathrm{O}}\right|$, symmetric $\mathrm{COO}$ stretch $R^{(\mathrm{s})}=\left(R_{\mathrm{C}-\mathrm{OH}}+R_{\mathrm{C}=\mathrm{O}}\right) / 2, \mathrm{CN}$ stretch $R^{(\mathrm{CN})}$, and $\mathrm{OH}$ stretch $R^{(\mathrm{OH})}$ vibrational coordinates.

acquires [(N2s) $\left.{ }^{-2}\right]$ character (cf. Fig. S2 [27]). At this point the system can either undergo a nonadiabatic transition to the PES of state $S_{118}$ or remain on the PES of $S_{119}$. A branching ratio of about $50 \%$ is found for this event as seen from the population of the two electronic states as a function of time shown in Fig. 4(b). Slower nuclear trajectories remain on the upper adiabatic PES, meaning that the holes evolve from the oxygen to the nitrogen atoms as the nuclei are displaced. Faster nuclear trajectories instead undergo a nonadiabatic transition between states $S_{119}$ and $S_{118}$ at the interaction region, which maintains the electronic wave function character. The carbon atoms bridge the double-hole transfer through the glycine backbone as seen by the involvement of configurations of $\left[(\mathrm{O} 2 s)^{-1},(\mathrm{C} 2 s)^{-1}\right]$ type in Fig. S2 for times of 3.5 and 6.0 fs [27].

In conclusion, we have theoretically described a mechanism for ultrafast double-hole transfer starting from $\left[\left(X 2 s^{-2}\right)\right]$ localized hole configurations that does not involve initial electronic coherences and have shown that these dynamics can be solely driven by small displacements of the nuclei of the order of a few tenths of an angstrom. These small atomic rearrangements occur before the onset of molecular fragmentation and are sufficient to affect the relative stability of the holes at the different molecular sites and to drive their evolution. The time scale involved is 3 to $4 \mathrm{fs}$, well below the shortest vibrational period of the molecule and comparable with the period for coherent hole migration typically reported for similar systems. Our finding could be relevant for x-ray imaging experiments, in which the ionization of inner atomic shells is unavoidable. It shows that small atomic displacements of valenceionized molecules can induce substantial shifts of charge density along the molecular skeleton, which we expect to affect the X-ray scattering patterns observed.

We thank the Hamburg Centre for Ultrafast Imaging for financial support, Ludger Inhester, Nikita Medvedev, LeePing Wang, and Basile Curchod for helpful discussions, and Todd Martínez for providing the ciopt program. Z. L. thanks the Volkswagen Foundation for support through a Paul-Ewald postdoctoral fellowship.

*zheng.li@cfel.de †oriol.vendrell@cfel.de

[1] C. A. Rozzi, S. M. Falke, N. Spallanzani, A. Rubio, E. Molinari, D. Brida, M. Maiuri, G. Cerullo, H. Schramm, J. Christoffers et al., Nat. Commun. 4, 1602 (2013).

[2] B. Erk et al., Science 345, 288 (2014).

[3] D. T. Ha, Y. Wang, M. Alcami, E. Itaelae, K. Kooser, S. Urpeainen, M. A. Huels, E. Kukk, and F. Martin, J. Phys. Chem. A 118, 1374 (2014).

[4] L. S. Cederbaum and J. Zobeley, Chem. Phys. Lett. 307, 205 (1999).

[5] J. Breidbach and L. S. Cederbaum, J. Chem. Phys. 118, 3983 (2003)

[6] J. Breidbach and L. S. Cederbaum, Phys. Rev. Lett. 94, 033901 (2005).

[7] F. Remacle and R. D. Levine, Proc. Natl. Acad. Sci. U.S.A. 103, 6793 (2006).

[8] A. I. Kuleff and L. S. Cederbaum, Phys. Rev. Lett. 106, 053001 (2011). 
[9] V. Despré, A. Marciniak, V. Loriot, M. C. E. Galbraith, A. Rouzée, M. J. J. Vrakking, F. Lépine, and A. I. Kuleff, J. Phys. Chem. Lett. 6, 426 (2015).

[10] B. Cooper and V. Averbukh, Phys. Rev. Lett. 111, 083004 (2013).

[11] J. Leeuwenburgh, B. Cooper, V. Averbukh, J. P. Marangos, and M. Ivanov, Phys. Rev. A 90, 033426 (2014).

[12] E. Goulielmakis, Z.-H. Loh, A. Wirth, R. Santra, N. Rohringer, V. S. Yakovlev, S. Zherebtsov, T. Pfeifer, A. M. Azzeer, M. F. Kling, S. R. Leone, and F. Krausz, Nature (London) 466, 739 (2010).

[13] H. Timmers, Z. Li, N. Shivaram, R. Santra, O. Vendrell, and A. Sandhu, Phys. Rev. Lett. 113, 113003 (2014).

[14] F. Calegari, D. Ayuso, A. Trabattoni, L. Belshaw, S. De Camillis, S. Anumula, F. Frassetto, L. Poletto, A. Palacios, P. Decleva, J. B. Greenwood, F. Martín, and M. Nisoli, Science 346, 336 (2014).

[15] R. Weinkauf, P. Schanen, D. Yang, S. Soukara, and E. W. Schlag, J. Phys. Chem. 99, 11255 (1995).

[16] Z. Li, Mohamed El-Amine Madjet, O. Vendrell, and R. Santra, Phys. Rev. Lett. 110, 038302 (2013).

[17] P. H. Bucksbaum, R. Coffee, and N. Berrah, in Advances in Atomic, Molecular and Optical Physics, Vol. 60, edited by E. Arimondo, P. R. Berman, and C. C. Lin (Academic Press is an imprint of Elsevier, New York, 2011), pp. 239-289.

[18] N. Berrah, L. Fang, B. Murphy, T. Osipov, K. Ueda, E. Kukk, R. Feifel, P. v. d. Meulen, P. Salen, H. T. Schmidt, R. D. Thomas, M. Larsson, R. Richter, K. C. Prince, J. D. Bozek, C. Bostedt, S.-i. Wada, M. N. Piancastelli, M. Tashiro, and M. Ehara, Proc. Natl. Acad. Sci. U.S.A. 108, 16912 (2011).

[19] J. Amann et al., Nat. Photonics 6, 693 (2012).

[20] J. Ullrich, A. Rudenko, and R. Moshammer, Annu. Rev. Phys. Chem. 63, 635 (2012).

[21] B. McFarland, J. Farrell, S. Miyabe, F. Tarantelli, A. Aguilar, N. Berrah, C. Bostedt, J. Bozek, P. Bucksbaum, J. Castagna et al., Nat. Commun. 5, 4235 (2014).

[22] L. Fang, T. Osipov, B. Murphy, A. Rudenko, D. Rolles, V. Petrovic, C. Bostedt, J. Bozek, P. Bucksbaum, and N. Berrah, J. Phys. B 47, 124006 (2014).

[23] H. Ågren, A. Cesar, and C.-M. Liegener, in Advances in Quantum Chemistry, Vol. 23, edited by P.-O. Löwdin, J. R. Sabin, and M. C. Zerner (Academic Press, New York, 1992), pp. 1-82.

[24] J. C. Tully, J. Chem. Phys. 93, 1061 (1990).

[25] Z. Li, M. E. Madjet, and O. Vendrell, J. Chem. Phys. 138, 094311 (2013).
[26] M. Barbatti, R. Shepard, and H. Lischka, in Conical Intersections: Theory, Computation and Experiment, edited by W. Domcke, D. R. Yarkony, and H. Köppel (World Scientific, Singapore, 2011), p. 415.

[27] See Supplemental Material at http://link.aps.org/ supplemental/10.1103/PhysRevLett.115.143002, which includes Refs. [28-35]. Details on the electronic structure and Auger spectra calculations and on the location of the relevant conical intersection.

[28] B. G. Levine, J. D. Coe, and T. J. Martínez, J. Phys. Chem. B 112, 405 (2008).

[29] I. D. Reva, A. M. Plokhotnichenko, S. G. Stepanian, A. Y. Ivanov, E. D. Radchenko, G. G. Sheina, and Y. P. Blagoi, Chem. Phys. Lett. 232, 141 (1995).

[30] E. Itälä, K. Kooser, E. Rachlew, M. A. Huels, and E. Kukk, J. Chem. Phys. 140, 234305 (2014).

[31] F. Aquilante, L. D. Vico, N. Feré, G. Ghigo, P. Å. Malmqvist, P. Neogrády, T. Pedersen, M. Pitonak, M. Reiher, B. Roos, L. Serrano-Andrés, M. Urban, V. Veryazov, and R. Lindh, J. Comput. Chem. 31, 224 (2010).

[32] V. Veryazov, P.-O. Widmark, L. Serrano-Andres, R. Lindh, and B. Roos, Int. J. Quantum Chem. 100, 626 (2004).

[33] G. Karlström, R. Lindh, P. Å. Malmqvist, B. O. Roos, U. Ryde, V. Veryazov, P.-O. Widmark, M. Cossi, B. Schimmelpfennig, P. Neogrády, and L. Seijo, Comput. Mater. Sci. 28, 222 (2003).

[34] R. Manne and H. Ågren, Chem. Phys. 93, 201 (1985).

[35] B. G. Levine, C. Ko, J. Quenneville, and T. J. Martínez, Mol. Phys. 104, 1039 (2006).

[36] D. Minelli, F. Tarantelli, A. Sgamellotti, and L. S. Cederbaum, J. Chem. Phys. 99, 6688 (1993).

[37] M. Mitani, O. Takahashi, K. Saito, and S. Iwata, J. Electron Spectrosc. Relat. Phenom. 128, 103 (2003).

[38] M. Tashiro, K. Ueda, and M. Ehara, J. Chem. Phys. 135, 154307 (2011)

[39] C.-M. Liegener, A. K. Bakhshi, R. Chen, and J. Ladik, J. Chem. Phys. 86, 6039 (1987).

[40] J. Eland, C. Rigby, E. Andersson, J. Palaudoux, L. Andric, F. Penent, P. Linusson, L. Hedin, L. Karlsson, J.-E. Rubensson et al., J. Chem. Phys. 132, 104311 (2010).

[41] J. Davies, J. LeClaire, R. Continetti, and C. Hayden, J. Chem. Phys. 111, 1 (1999).

[42] A. Vredenborg, W. G. Roeterdink, and M. H. Janssen, J. Chem. Phys. 128, 204311 (2008).

[43] N. Gador, E. Samoylova, V. R. Smith, A. Stolow, D. M. Rayner, W. Radloff, I. V. Hertel, and T. Schultz, J. Phys. Chem. A 111, 11743 (2007).

[44] R. S. Mulliken, J. Chem. Phys. 23, 1833 (1955). 